\title{
On Iyengar-type inequalities via quasi-convexity and quasi-concavity
}

\author{
M. Emin Özdemir
}




\title{
ON IYENGAR-TYPE INEQUALITIES VIA QUASI-CONVEXITY AND QUASI-CONCAVITY
}

\author{
M. EMIN ÖZDEMIR
}

Received 30 November, 2012

\begin{abstract}
In this paper, we obtain some new estimations of Iyengar-type inequality in which quasi-convex(quasi-concave) functions are involved. These estimations are improvements of some recently obtained estimations. Some error estimations for the trapezoidal formula are given. Applications for special means are also provided.
\end{abstract}

2010 Mathematics Subject Classification: 26D15

Keywords: weighted Hölder inequality, Hölder inequality, power-mean inequality, differentiable function, quasi-convex function

\section{INTRODUCTION}

If it is necessary to bound one quantity by another, the classical inequalities are very useful for this purpose. This first book called "Inequalities" written by Hardy, Littlewood and Polya at Cambridge University Press in 1934 represents the first effort to systemize a rapidly expanding domain. In this sense, the second important book "Classical and New Inequalities in Analysis" is written by D.S. Mitrinović, J.E.Pećarič and A.M. Fink. The third book "Analytic Inequalities" is written by D.S. Mitrinović, and the other book "Means and Their Inequalities" is written by Bullen, D.S. Mitrinović, D.S. Vasic, P.M.

Today inequalities play a significant role in the development in all fields of Mathematics. They have applications in a variety of applied Mathematics. For example, convex functions are tractable in optimization because local optimality guarantees global optimality. In recent years a number of authors have discovered new integral inequalities for convex, $s$-convex, logarithmic convex, $h$-convex, quasi-convex, $m$-convex, $(\alpha, m)$-convex, co-ordinated convex, Godunova-Levin and $P$-functions.

On November 22, 1881, Hermite (1822-1901) sent a letter to the Journal Mathesis. This letter was published in Mathesis 3 (1883,p.82) and in this letter an inequality presented which is well-known in the literature as Hermite-Hadamard integral 
inequality :

$$
f\left(\frac{a+b}{2}\right) \leq \frac{1}{b-a} \int_{a}^{b} f(x) d x \leq \frac{f(a)+f(b)}{2},
$$

where $f: I \subseteq \mathbb{R} \rightarrow \mathbb{R}$ is a convex function on the interval $I$ of a real numbers and $a, b \in I$ with $a<b$. If the function $f$ is concave, the inequality in (1.1) is reversed. That is

$$
f\left(\frac{a+b}{2}\right) \geq \frac{1}{b-a} \int_{a}^{b} f(x) d x \geq \frac{f(a)+f(b)}{2} .
$$

For recent results, generalizations and new inequalities related to the inequality (1.1) see $([1,3-5,7,8,10-15,18-23])$.

Then left hand side of Hermite-Hadamard inequality $(\mathrm{LHH})$ can also be estimated by the inequality of Iyengar.

$$
\frac{f(a)+f(b)}{2}-\frac{1}{b-a} \int_{a}^{b} f(x) d x \leq \frac{M(b-a)}{4}-\frac{[f(b)-f(a)]^{2}}{4 M(b-a)}
$$

where

$$
M=\sup \left\{\left|\frac{f(x)-f(y)}{x-y}\right| ; x \neq y\right\}
$$

In [9], Daniel Alexandru Ion proved the following inequalities of Iyengar type for differentiable quasi-convex functions:

$$
\left|\frac{f(a)+f(b)}{2}-\frac{1}{b-a} \int_{a}^{b} f(x) d x\right| \leq \frac{(b-a)}{4}\left(\sup \left\{\left|f^{\prime}(a)\right|,\left|f^{\prime}(b)\right|\right\}\right)
$$

where $f:[a, b] \rightarrow \mathbb{R}$ is differentiable function on $(a, b)$, and $\left|f^{\prime}\right|$ is quasi-convex on $[a, b]$ with $a<b$.

and

$$
\begin{aligned}
& \left|\frac{f(a)+f(b)}{2}-\frac{1}{b-a} \int_{a}^{b} f(x) d x\right| \\
\leq & \frac{(b-a)}{2(p+1)^{\frac{1}{p}}}\left(\sup \left\{\left|f^{\prime}(a)\right|^{\frac{p}{p-1}},\left|f^{\prime}(b)\right|^{\frac{p}{p-1}}\right\}\right)^{\frac{p-1}{p}}
\end{aligned}
$$

where $f:[a, b] \rightarrow \mathbb{R}$ is differentiable function on $(a, b)$, and $\left|f^{\prime}\right|^{\frac{p}{p-1}}$ is quasi-convex on $[a, b]$ with $a<b$.

We give some necessary definitions and mathematical preliminaries for quasi-convex functions which are used throughout this paper.

Definition 1 (see [16]). A function $f:[a, b] \rightarrow \mathbb{R}$ is said to be quasi-convex on $[a, b]$ if

$$
f(\lambda x+(1-\lambda) y) \leq \max \{f(x), f(y)\},
$$


holds for all $x, y \in[a, b]$ and $\lambda \in[0,1]$. For additional results on quasi-convexity, see [17]. Clearly, any convex function is quasi-convex function. Furthermore, there exists quasi-convex functions which are not convex. See [9] :

$$
g(t)= \begin{cases}1, & t \in[-2,-1] \\ t^{2}, & t \in(-1,2]\end{cases}
$$

is not a convex function on $[-2,2]$, but it is a quasi-convex function on [-2, 2].If we choose $g:[-2,2] \rightarrow \mathbb{R}, g(-2)=1, g(2)=4$ and for $\alpha=\frac{1}{2}, a=-2, b=0$, we get $g(\alpha a+(1-\alpha) b)=g(-1)=1$ and $\alpha g(a)+(1-\alpha) g(b)=\frac{1}{2} g(-2)+\frac{1}{2} g(0)=\frac{1}{2}$. Thus it is not convex but it is quasi-convex function for all $\alpha \in[0,1]$,

$$
g(-\alpha 2+(1-\alpha) 2) \leq \max \{g(-2), g(2)\}=\max \{1,4\}=4 .
$$

The main purpose of this paper is to point out new estimations of the inequality in (1.2), but now for the class of quasi-convex functions.

In order to prove our main results we need the following lemma (see [2]).

Lemma 1. Let $f: I \subset \mathbb{R} \rightarrow \mathbb{R}$ be a twice differentiable mapping on $I^{\circ}, a, b \in I$ with $a<b$ and $f^{\prime \prime}$ be integrable on $[a, b]$. Then the following equality holds:

$$
\frac{f(a)+f(b)}{2}-\frac{1}{b-a} \int_{a}^{b} f(x) d x=\frac{(b-a)^{2}}{2} \int_{0}^{1} t(1-t) f^{\prime \prime}(t a+(1-t) b) d t .
$$

The main results of this paper are given by the following theorems.

\section{Results}

Theorem 1. Let $f: I^{\circ} \subset[0, \infty) \rightarrow \mathbb{R}$, be a twice differentiable mapping on $I^{\circ}$, such that $f^{\prime \prime} \in L[a, b], a, b \in I$ with $a<b$. If $\left|f^{\prime \prime}\right|^{q}$ is quasi-convex on $[a, b]$ for $q>1$, then the following inequality holds:

$$
\begin{aligned}
& \left|\frac{f(a)+f(b)}{2}-\frac{1}{b-a} \int_{a}^{b} f(x) d x\right| \\
\leq & \frac{(b-a)^{2}}{2}\left(\frac{q-1}{2 q-p-1}\right)^{\frac{q-1}{q}}(\beta(p+1, q+1))^{\frac{1}{q}} \\
& \times\left(\max \left\{\left|f^{\prime \prime}(a)\right|^{q},\left|f^{\prime \prime}(b)\right|^{q}\right\}\right)^{\frac{1}{q}}
\end{aligned}
$$

where $\frac{1}{p}+\frac{1}{q}=1$ and $\beta($, ) is Euler Beta Function:

$$
\beta(x, y)=\int_{0}^{1} t^{x-1}(1-t)^{y-1} d t, \quad x, y>0 .
$$


Theorem 2. Let $f: I^{\circ} \subset[0, \infty) \rightarrow \mathbb{R}$, be a twice differentiable mapping on $I^{\circ}$, such that $f^{\prime \prime} \in L[a, b], a, b \in I$ with $a<b$. If $\left|f^{\prime \prime}\right|^{q}$ is quasi-convex on $[a, b]$ for $q \geq 1$, then the following inequality holds:

$$
\begin{aligned}
& \left|\frac{f(a)+f(b)}{2}-\frac{1}{b-a} \int_{a}^{b} f(x) d x\right| \\
\leq & \frac{(b-a)^{2}}{4}\left(\frac{2}{(q+1)(q+2)}\right)^{\frac{q-1}{q}}\left(\max \left\{\left|f^{\prime \prime}(a)\right|^{q},\left|f^{\prime \prime}(b)\right|^{q}\right\}\right)^{\frac{1}{q}} .
\end{aligned}
$$

Theorem 3. With the assumptions of Theorem 1, we obtain another

$$
\begin{aligned}
& \left|\frac{f(a)+f(b)}{2}-\frac{1}{b-a} \int_{a}^{b} f(x) d x\right| \\
\leq & \frac{(b-a)^{2}}{2^{1+\frac{1}{q}}}(\beta(2, p+1))^{\frac{1}{p}}\left(\max \left\{\left|f^{\prime \prime}(a)\right|^{q},\left|f^{\prime \prime}(b)\right|^{q}\right\}\right)^{\frac{1}{q}} .
\end{aligned}
$$

Proof of Theorem 1. Using Lemma 1 and the well known Hölder's inequality for $q>1$,

$$
\begin{aligned}
& \left|\frac{f(a)+f(b)}{2}-\frac{1}{b-a} \int_{a}^{b} f(x) d x\right| \\
\leq & \frac{(b-a)^{2}}{2}\left(\int_{0}^{1} t^{\frac{q-p}{q-1}} d t\right)^{\frac{q-1}{q}}\left[\int_{0}^{1} t^{p}(1-t)^{q}\left|f^{\prime \prime}(t a+(1-t) b)\right|^{q} d t\right]^{\frac{1}{q}},
\end{aligned}
$$

where $\frac{1}{p}+\frac{1}{q}=1$.

On the other hand, since $\left|f^{\prime \prime}\right|^{q}$ is quasi-convex on $[a, b]$, we know that for any $t \in[0,1]$

$$
\left|f^{\prime \prime}(t a+(1-t) b)\right|^{q} \leq \max \left\{\left|f^{\prime \prime}(a)\right|^{q},\left|f^{\prime \prime}(b)\right|^{q}\right\} .
$$

Therefore, we obtain

$$
\begin{aligned}
& \left|\frac{f(a)+f(b)}{2}-\frac{1}{b-a} \int_{a}^{b} f(x) d x\right| \\
\leq & \frac{(b-a)^{2}}{2}\left(\int_{0}^{1} t^{\frac{q-p}{q-1}} d t\right)^{\frac{q-1}{q}}\left[\int_{0}^{1} t^{p}(1-t)^{q}\left|f^{\prime \prime}(t a+(1-t) b)\right|^{q} d t\right]^{\frac{1}{q}} \\
\leq & \frac{(b-a)^{2}}{2}\left(\int_{0}^{1} t^{\frac{q-p}{q-1}} d t\right)^{\frac{q-1}{q}}\left[\int_{0}^{1} t^{p}(1-t)^{q}\left(\max \left\{\left|f^{\prime \prime}(a)\right|^{q},\left|f^{\prime \prime}(b)\right|^{q}\right\}\right) d t\right]^{\frac{1}{q}} \\
= & \frac{(b-a)^{2}}{2}\left(\frac{q-1}{2 q-p-1}\right)^{\frac{q-1}{q}}(\beta(p+1, q+1))^{\frac{1}{q}}\left(\max \left\{\left|f^{\prime \prime}(a)\right|^{q},\left|f^{\prime \prime}(b)\right|^{q}\right\}\right)^{\frac{1}{q}},
\end{aligned}
$$


which completes the proof.

Corollary 1. In Theorem 1, if we choose $M=\operatorname{Sup}_{x \in(a, b)}\left|f^{\prime \prime}(x)\right|<\infty$, we get

$$
\begin{aligned}
& \left|\frac{f(a)+f(b)}{2}-\frac{1}{b-a} \int_{a}^{b} f(x) d x\right| \\
\leq & \frac{(b-a)^{2}}{2} M\left(\frac{q-1}{2 q-p-1}\right)^{\frac{q-1}{q}}(\beta(p+1, q+1))^{\frac{1}{q}} .
\end{aligned}
$$

Proof of Theorem 2. From Lemma 1 and the well known power-mean inequality we obtain

$$
\begin{aligned}
& \left|\frac{f(a)+f(b)}{2}-\frac{1}{b-a} \int_{a}^{b} f(x) d x\right| \\
\leq & \frac{(b-a)^{2}}{2} \int_{0}^{1} t(1-t)\left|f^{\prime \prime}(t a+(1-t) b)\right| d t \\
\leq & \frac{(b-a)^{2}}{2}\left(\int_{0}^{1} t d t\right)^{1-\frac{1}{q}}\left(\int_{0}^{1} t(1-t)^{q}\left|f^{\prime \prime}(t a+(1-t) b)\right|^{q} d t\right)^{\frac{1}{q}} \\
\leq & \frac{(b-a)^{2}}{2}\left(\int_{0}^{1} t d t\right)^{1-\frac{1}{q}}\left(\int_{0}^{1} t(1-t)^{q}\left(\max \left\{\left|f^{\prime \prime}(a)\right|^{q},\left|f^{\prime \prime}(b)\right|^{q}\right\}\right) d t\right)^{\frac{1}{q}} \\
= & \frac{(b-a)^{2}}{2}\left(\frac{1}{2}\right)^{1-\frac{1}{q}}\left(\frac{1}{(q+1)(q+2)}\right)^{\frac{1}{q}}\left(\max \left\{\left|f^{\prime \prime}(a)\right|^{q},\left|f^{\prime \prime}(b)\right|^{q}\right\}\right)^{\frac{1}{q}} \\
= & \frac{(b-a)^{2}}{4}\left(\frac{2}{(q+1)(q+2)}\right)^{\frac{1}{q}}\left(\max \left\{\left|f^{\prime \prime}(a)\right|^{q},\left|f^{\prime \prime}(b)\right|^{q}\right\}\right)^{\frac{1}{q}} \cdot
\end{aligned}
$$

The proof of Theorem 2 is completed.

Corollary 2. Under the assumptions of Theorem 2,

Case i: Since $\lim _{q \rightarrow \infty}\left(\frac{2}{(q+1)(q+2)}\right)^{\frac{1}{q}}=1$ and $\lim _{q \rightarrow 1^{+}}\left(\frac{2}{(q+1)(q+2)}\right)^{\frac{1}{q}}=\frac{1}{3}$, we have

$$
\frac{1}{3}<\left(\frac{2}{(q+1)(q+2)}\right)^{\frac{1}{q}}<1, \quad q \in[1, \infty) .
$$

Therefore,

$$
\left|\frac{f(a)+f(b)}{2}-\frac{1}{b-a} \int_{a}^{b} f(x) d x\right| \leq \frac{(b-a)^{2}}{4}\left(\max \left\{\left|f^{\prime \prime}(a)\right|^{q},\left|f^{\prime \prime}(b)\right|^{q}\right\}\right)^{\frac{1}{q}} .
$$

$\operatorname{In}(2.3)$, 
- if $\left|f^{\prime \prime}\right|^{q}$ is decreasing, we get

$$
\left|\frac{f(a)+f(b)}{2}-\frac{1}{b-a} \int_{a}^{b} f(x) d x\right| \leq \frac{(b-a)^{2}}{4}\left|f^{\prime \prime}(a)\right|,
$$

- if $\left|f^{\prime \prime}\right|^{q}$ is increasing, we get

$$
\left|\frac{f(a)+f(b)}{2}-\frac{1}{b-a} \int_{a}^{b} f(x) d x\right| \leq \frac{(b-a)^{2}}{4}\left|f^{\prime \prime}(b)\right| .
$$

Case ii: If we choose $M=\operatorname{Sup}_{x \in(a, b)}\left|f^{\prime \prime}(x)\right|<\infty$ in (2.2), then the inequality in (2.1) is better than the inequality in (2.2).

Proof of Theorem 3. From Lemma 1 with properties of modulus we get

$$
\begin{aligned}
& \left|\frac{f(a)+f(b)}{2}-\frac{1}{b-a} \int_{a}^{b} f(x) d x\right| \\
\leq & \frac{(b-a)^{2}}{2} \int_{0}^{1} t(1-t)\left|f^{\prime \prime}(t a+(1-t) b)\right| d t .
\end{aligned}
$$

Now, if we use the following weighted version of Hölder's inequality [6, p. 117]:

$$
\left|\int_{I} f(s) g(s) h(s) d s\right| \leq\left(\int_{I}|f(s)|^{p} h(s) d s\right)^{\frac{1}{p}}\left(\int_{I}|g(s)|^{q} h(s) d s\right)^{\frac{1}{q}}
$$

for $p>1, p^{-1}+q^{-1}=1, h$ is nonnegative on $I$ and provided all the other integrals exist and are finite.

If we rewrite the inequality (2.4) with respect to (2.5) with $\left|f^{\prime \prime}\right|^{q}$ is quasi-convex on $[a, b]$ for all $t \in[0,1]$, we get

$$
\begin{aligned}
& \left|\frac{f(a)+f(b)}{2}-\frac{1}{b-a} \int_{a}^{b} f(x) d x\right| \\
\leq & \frac{(b-a)^{2}}{2} \int_{0}^{1} t(1-t)\left|f^{\prime \prime}(t a+(1-t) b)\right| d t \\
= & \frac{(b-a)^{2}}{2} \int_{0}^{1}(1-t)\left|f^{\prime \prime}(t a+(1-t) b)\right| t d t \\
\leq & \frac{(b-a)^{2}}{2}\left(\int_{0}^{1}(1-t)^{p} t d t\right)^{\frac{1}{p}}\left(\int_{0}^{1}\left|f^{\prime \prime}(t a+(1-t) b)\right|^{q} t d t\right)^{\frac{1}{q}} \\
= & \frac{(b-a)^{2}}{2}(\beta(2, p+1))^{\frac{1}{p}}\left(\frac{\max \left\{\left|f^{\prime \prime}(a)\right|^{q},\left|f^{\prime \prime}(b)\right|^{q}\right\}}{2}\right)^{\frac{1}{q}} .
\end{aligned}
$$

The proof of Theorem 3 is completed. 
Corollary 3. In Theorem 3, if we choose $M=S u p_{x \in(a, b)}\left|f^{\prime \prime}(x)\right|<\infty$, we get

$$
\left|\frac{f(a)+f(b)}{2}-\frac{1}{b-a} \int_{a}^{b} f(x) d x\right| \leq \frac{(b-a)^{2}}{2^{1+\frac{1}{q}}} M(\beta(2, p+1))^{\frac{1}{p}} .
$$

Remark 1. From Theorems 1-3, we get

$$
\left|\frac{f(a)+f(b)}{2}-\frac{1}{b-a} \int_{a}^{b} f(x) d x\right| \leq \min \left\{v_{1}, v_{2}, v_{3}\right\}
$$

where

$$
\begin{gathered}
v_{1}= \\
\frac{(b-a)^{2}}{2}\left(\frac{q-1}{2 q-p-1}\right)^{\frac{q-1}{q}}(\beta(p+1, q+1))^{\frac{1}{q}} \times\left(\max \left\{\left|f^{\prime \prime}(a)\right|^{q},\left|f^{\prime \prime}(b)\right|^{q}\right\}\right)^{\frac{1}{q}} \\
v_{2}=\frac{(b-a)^{2}}{4}\left(\frac{2}{(q+1)(q+2)}\right)^{\frac{q-1}{q}}\left(\max \left\{\left|f^{\prime \prime}(a)\right|^{q},\left|f^{\prime \prime}(b)\right|^{q}\right\}\right)^{\frac{1}{q}}
\end{gathered}
$$

and

$$
v_{3}=\frac{(b-a)^{2}}{2^{1+\frac{1}{q}}}(\beta(2, p+1))^{\frac{1}{p}}\left(\max \left\{\left|f^{\prime \prime}(a)\right|^{q},\left|f^{\prime \prime}(b)\right|^{q}\right\}\right)^{\frac{1}{q}} .
$$

3. ERror Estimates For the TrapezoidAl RULE

Let $d$ be a partition $a=x_{0}<x_{1}<x_{2}<\ldots<x_{n}=b$ of the interval $[a, b]$ and consider the quadrature formula

$$
\int_{a}^{b} f(x) d x=T_{i}(f, d)+E_{i}(f, d), \quad i=1,2, \ldots, n-1
$$

where

$$
T_{1}(f, d)=\sum_{i=0}^{n-1} \frac{f\left(x_{i}\right)+f\left(x_{i+1}\right)}{2}\left(x_{i+1}-x_{i}\right)
$$

for the Trapezoidal version and

$$
T_{2}(f, d)=\sum_{i=0}^{n-1} f\left(\frac{x_{i}+x_{i+1}}{2}\right)\left(x_{i+1}-x_{i}\right)
$$

for the Midpoint formula and $E_{i}(f, d)$ denotes the associated approximation errors.

Proposition 1. Suppose that all the assumptions of Theorem 1 are satisfied for every division $d$ of $[a, b]$, we have

$$
|E(f, d)| \leq \frac{1}{2}\left(\frac{q-1}{2 q-p-1}\right)^{\frac{q-1}{q}}(\beta(p+1, q+1))^{\frac{1}{q}}
$$




$$
\times \sum_{i=0}^{n-1}\left(x_{i+1}-x_{i}\right)^{3}\left(\max \left\{\left|f^{\prime \prime}\left(x_{i}\right)\right|^{q},\left|f^{\prime \prime}\left(x_{i+1}\right)\right|^{q}\right\}\right)^{\frac{1}{q}} .
$$

Proof. Applying Theorem 1 on the subinterval $\left(x_{i+1}, x_{i}\right), i=1,2, \ldots, n-1$ of the partition and by using the quasi-convexity of $\left|f^{\prime \prime}\right|^{q}$, we obtain

$$
\begin{aligned}
& \left|\frac{f\left(x_{i}\right)+f\left(x_{i+1}\right)}{2}-\frac{1}{\left(x_{i+1}-x_{i}\right)} \int_{x_{i}}^{x_{i+1}} f(x) d x\right| \\
\leq & \frac{\left(x_{i+1}-x_{i}\right)^{2}}{2}\left(\frac{q-1}{2 q-p-1}\right)^{\frac{q-1}{q}}(\beta(p+1, q+1))^{\frac{1}{q}} \\
& \times\left(\max \left\{\left|f^{\prime \prime}\left(x_{i}\right)\right|^{q},\left|f^{\prime \prime}\left(x_{i+1}\right)\right|^{q}\right\}\right)^{\frac{1}{q}} .
\end{aligned}
$$

Hence in (3.1), we have

$$
\begin{aligned}
\left|\int_{a}^{b} f(x) d x-T(f, d)\right| & =\left|\sum_{i=0}^{n-1}\left\{\int_{x_{i}}^{x_{i}+1} f(x) d x-\frac{f\left(x_{i}\right)+f\left(x_{i+1}\right)}{2}\left(x_{i+1}-x_{i}\right)\right\}\right| \\
& \leq \sum_{i=0}^{n-1}\left|\int_{x_{i}}^{x_{i+1}} f(x) d x-\frac{f\left(x_{i}\right)+f\left(x_{i+1}\right)}{2}\left(x_{i+1}-x_{i}\right)\right| \\
& \leq \frac{1}{2}\left(\frac{q-1}{2 q-p-1}\right)^{\frac{q-1}{q}}(\beta(p+1, q+1))^{\frac{1}{q}} \\
& \times \sum_{i=0}^{n-1}\left(x_{i+1}-x_{i}\right)^{3}\left(\max \left\{\left|f^{\prime \prime}\left(x_{i}\right)\right|^{q},\left|f^{\prime \prime}\left(x_{i+1}\right)\right|^{q}\right\}\right)^{\frac{1}{q}} .
\end{aligned}
$$

Proposition 2. Suppose that all the assumptions of Theorem 2 are satisfied for every division d of $[a, b]$, we have

$$
\begin{aligned}
|E(f, d)| \leq & \frac{1}{4}\left(\frac{2}{(q+1)(q+2)}\right)^{\frac{1}{q}} \\
& \times \sum_{i=0}^{n-1}\left(x_{i+1}-x_{i}\right)^{3}\left(\max \left\{\left|f^{\prime \prime}\left(x_{i}\right)\right|^{q},\left|f^{\prime \prime}\left(x_{i+1}\right)\right|^{q}\right\}\right)^{\frac{1}{q}} .
\end{aligned}
$$

Proof. The proof immediately follows from Theorem 2 and by applying a similar argument to Proposition 1.

Proposition 3. Suppose that all the assumptions of Theorem 3 are satisfied for every division $d$ of $[a, b]$, we have

$$
|E(f, d)| \leq \frac{1}{2}(\beta(1, p+1))^{\frac{1}{p}}
$$




$$
\times \sum_{i=0}^{n-1}\left(x_{i+1}-x_{i}\right)^{2}\left(\frac{\max \left\{\left|f^{\prime \prime}(a)\right|^{q},\left|f^{\prime \prime}(b)\right|^{q}\right\}}{2}\right)^{\frac{1}{q}} .
$$

Proof. The proof immediately follows from Theorem 3 and by applying a similar argument to Proposition 1.

\section{Applications to SPECial MEANS}

Let us consider the special means for real numbers $a, b(a \neq b)$. We take

1. Arithmetic mean:

$$
A(a, b)=\frac{a+b}{2}, \quad a, b \in \mathbb{R} .
$$

\section{Logarithmic mean:}

$$
L(a, b)=\frac{a-b}{\ln |a|-\ln |b|}, \quad|a| \neq|b|, a, b \neq 0, a, b \in \mathbb{R} .
$$

3. Generalized log-mean:

$$
L_{n}(a, b)=\left[\frac{b^{n+1}-a^{n+1}}{(n+1)(b-a)}\right]^{\frac{1}{n}}, \quad n \in \mathbb{Z} \backslash\{-1,0\}, a, b \in \mathbb{R}, a \neq b .
$$

Proposition 4. Let $a, b \in \mathbb{R}, a<b$ and $n \in \mathbb{N}, n \geq 2$. Then we have

$$
\begin{aligned}
& \left|A\left(a^{n}, b^{n}\right)-L_{n}^{n}(a, b)\right| \leq \frac{n(n-1)(b-a)^{2}}{2} \\
& \times\left(\frac{q-1}{2 q-p-1}\right)^{\frac{q-1}{q}}(\beta(p+1, q+1))^{\frac{1}{q}}\left(\max \left\{|a|^{(n-2) q},|b|^{(n-2) q}\right\}\right)^{\frac{1}{q}} .
\end{aligned}
$$

Proof. The assertion follows from Theorem 1 applied to the quasi-convex mapping $f(x)=x^{n}, x \in \mathbb{R}$.

Proposition 5. Let $a, b \in \mathbb{R}, a<b$ and $n \in \mathbb{N}, n \geq 2$. Then we have

$$
\begin{aligned}
& \left|A\left(a^{n}, b^{n}\right)-L_{n}^{n}(a, b)\right| \leq \frac{n(n-1)(b-a)^{2}}{4} \\
& \times\left(\frac{2}{(q+1)(q+2)}\right)^{\frac{1}{q}}\left(\max \left\{|a|^{(n-2) q},|b|^{(n-2) q}\right\}\right)^{\frac{1}{q}} .
\end{aligned}
$$

Proof. The assertion follows from Theorem 2 applied to the quasi-convex mapping $f(x)=x^{n}, x \in \mathbb{R}$.

Proposition 6. Let $a, b \in \mathbb{R}, a<b$ and $n \in \mathbb{N}, n \geq 2$. Then we have

$$
\left|A\left(a^{n}, b^{n}\right)-L_{n}^{n}(a, b)\right| \leq \frac{n(n-1)(b-a)^{2}}{2^{1+\frac{1}{q}}}
$$




$$
\times(\beta(2, q+1))^{\frac{1}{p}}\left(\max \left\{|a|^{(n-2) q},|b|^{(n-2) q}\right\}\right)^{\frac{1}{q}} .
$$

Proof. The assertion follows from Theorem 3 applied to the quasi-convex mapping $f(x)=x^{n}, x \in \mathbb{R}$.

\section{REFERENCES}

[1] A. O. Akdemir, M. E. Özdemir, and S. Varošanec, "On some inequalities for $h$-concave functions," Math. Comput. Modelling, vol. 55, no. 3-4, pp. 746-753, 2012.

[2] M. Alomari, M. Darus, and S. S. Dragomir, "New inequalities of Hermite- Hadamard type for functions whose second derivatives absolute value are quasi-convex," RGMIA Res. Rep. Coll., vol. 12, p. Article 17, 2009.

[3] M. Avci, H. Kavurmaci, and M. E. Özdemir, "New inequalities of Hermite-Hadamard type via $s$-convex functions in the second sense with applications," Appl. Math. Comput., vol. 217, no. 12, pp. 5171-5176, 2011.

[4] M. K. Bakula, U. S. Kirmaci, M. E. Özdemir, and J. Pečarić, "Hadamard-type inequalities for s-convex functions," Appl. Math. Comput., vol. 193, no. 1, pp. 26-35, 2007.

[5] M. K. Bakula, M. E. Özdemir, and J. E. Pecaric, "Hadamard type inequalities for $m$-convex and ( $\alpha, m)$-convex functions," JIPAM, J. Inequal. Pure Appl. Math., vol. 9, no. 4, p. 12, 2008.

[6] S. S. Dragomir, R. P. Agarwal, and N. S. Barnett, "Inequalities for Beta and Gamma functions via some classical and new integral inequalities," J. Inequal. Appl., vol. 5, no. 2, pp. 103-165, 2000.

[7] A. Házy, "Bernstein-Doetsch type results for $h$-convex functions," Math. Inequal. Appl., vol. 14, no. 3, pp. 499-508, 2011.

[8] A. Házy, "Bernstein-Doetsch type results for $(k, h)$-convex functions," Miskolc Math. Notes, vol. 13, no. 2, pp. 325-336, 2012.

[9] D. A. Ion, "Some estimates on the Hermite-Hadamard inequality through quasi-convex functions," An. Univ. Craiova, Ser. Mat. Inf., vol. 34, pp. 83-88, 2007.

[10] H. Kavurmaci, M. Avci, and M. E. Özdemir, "New inequalities of Hermite-Hadamard type for convex functions with applications," J. Inequal. Appl., vol. 2011, p. 11, 2011.

[11] B. Micherda and T. Rajba, "On some Hermite-Hadamard-Fejér inequalities for $(k, h)$-convex functions," Math. Inequal. Appl., vol. 15, no. 4, pp. 931-940, 2012

[12] M. E. Özdemir and A. O. Akdemir, "On some hadamard type inequalities for convex functions on a rectangular box," Journal of nonlinear Analysis and Application, vol. 2011, no. Article ID jnaa-00101, p. 10, 2011.

[13] M. E. Özdemir, c. Yildiz, and A. O. Akdemir, "On some new Hadamard type inequalities for coordinated quasi-convex functions," submitted.

[14] M. E. Özdemir, M. Avci, and H. Kavurmaci, "Hermite-Hadamard-type inequalities via $(\alpha, m)$ convexity," Comput. Math. Appl., vol. 61, no. 9, pp. 2614-2620, 2011.

[15] M. E. Özdemir, M. Avcı, and E. Set, "On some inequalities of Hermite-Hadamard type via $m$ convexity," Appl. Math. Lett., vol. 23, no. 9, pp. 1065-1070, 2010.

[16] J. E. Pečarić, F. Proschan, and Y. Tong, Convex functions, partial orderings, and statistical applications., ser. Mathematics in Science and Engineering. Boston, MA: Academic Press, 1992, vol. 187.

[17] J. Ponstein, "Seven kinds of convexity," SIAM Rev., vol. 9, pp. 115-119, 1967.

[18] M. Z. Sarikaya, A. Saglam, and H. Yildirim, "On some Hadamard-type inequalities for $h$-convex functions," J. Math. Inequal., vol. 2, no. 3, pp. 335-341, 2008.

[19] E. Set, M. E. Özdemir, and S. S. Dragomir, "On the Hermite-Hadamard inequality and other integral inequalities involving two functions," J. Inequal. Appl., vol. 2010, p. 9, 2010. 
[20] E. Set, M. Özdemir, and S. S. Dragomir, "On Hadamard-type inequalities involving several kinds of convexity," J. Inequal. Appl., vol. 2010, p. 12, 2010.

[21] M. Tunç, "On some integral inequalities via h-convexity," Miskolc Math. Notes, vol. 14, no. 3, pp. 1041-1057, 2013.

[22] M. Tunç, "Ostrowski-type inequalities via $h$-convex functions with applications to special means," J. Inequal. Appl., vol. 2013, p. 10, 2013.

[23] S. Varošanec, "On h-convexity," J. Math. Anal. Appl., vol. 326, no. 1, pp. 303-311, 2007.

\section{Author's address}

M. Emin Özdemir

Atatürk University, K.K. Education Faculty, Department of Mathematics, 25240, Campus, Erzurum, Turkey

E-mail address: emoseatauni.edu.tr 